\title{
Consideraciones perioperatorias en el paciente obeso
}

\author{
Claudio Nazar J. ${ }^{1}$, Roberto Coloma D. ${ }^{1}$, José Ignacio Contreras C. ${ }^{1}$, \\ Ian Molina P. ${ }^{1}$ y Ricardo Fuentes H. ${ }^{1}$
}

'Escuela de Medicina, Facultad de Medicina, Pontificia Universidad Católica de Chile. Santiago, Chile.

Recibido el 11 de noviembre de 2017, aceptado para publicación el 26 de marzo de 2018 .

Correspondencia a: Dr. Ricardo Fuentes $\mathrm{H}$. rfuente@med.puc.c

\section{Perioperative considerations in the obese patient}

Obesity is a condition of excessive accumulation of body fat to a level that is harmful to health. The prevalence of obesity is increasing worldwide at an alarming rate. Therefore it is essential to know the perioperative management and specific considerations in this population. The majority of obese patients who undergo surgical procedures have few additional pathology, so their perioperative risk is similar to eutrophic patients. Obesity Surgery Mortality Risk Stratification Score OS-MRS is a validated scale for assessing the risk of perioperative mortality in these patients. It is essential to look for the presence of respiratory sleep disorders in these patients, and for this purpose, the STOP-BANG questionnaire is the most valid today. During anesthetic induction it is recommended to place the patient in the "ramp" position as it significantly improves the visualization of the glottis during laryngoscopy. Also, this position optimizes the oxygenation and ventilatory mechanics of these patients, increasing the time of safe apnea. Regarding airway management, orotracheal intubation with controlled ventilation is the technique of choice in this population. Anesthetic drug doses should be calculated based on adjusted weight or lean weight, depending on the type of drug chosen. Finally, complete and frequent postoperative monitoring is recommended in the recovery unit, transferring these patients to their room only when they meet the criteria included in the White and Song scale.

Key words: obesity; perioperative; surgery; considerations.

\section{Resumen}

La obesidad es una condición de acumulación excesiva de grasa corporal a un nivel que resulta dañino para la salud. La prevalencia de obesidad está aumentando en todo el mundo a un ritmo alarmante, por lo tanto, es fundamental conocer el manejo perioperatorio y consideraciones específicas en esta población. La mayoría de los pacientes obesos que se someten a procedimientos quirúrgicos tienen pocas patologías agregadas, por lo que su riesgo perioperatorio es similar a pacientes normopesos. El Obesity Surgery Mortality Risk Stratification Score OS-MRS es una escala validada para evaluar el riesgo de mortalidad perioperatoria en estos pacientes. Es esencial buscar la presencia de trastornos respiratorios del sueño en estos pacientes, y para ello el cuestionario $S T O P-B A N G$ es el de mayor validez en la actualidad. Durante la inducción anestésica se recomienda colocar al paciente en posición "de rampa" ya que mejora significativamente la visualización de la glotis durante la laringoscopia. Además, esta posición optimiza la oxigenación y mecánica ventilatoria de estos pacientes, aumentando el tiempo de apnea seguro. Respecto al manejo de vía aérea, la intubación orotraqueal con ventilación controlada es la técnica de elección en esta población. Las dosis de drogas anestésicas deben calcularse en base al peso ajustado o magro, dependiendo del tipo de fármaco elegido. Finalmente, se recomienda una monitorización posoperatoria completa y frecuente en la unidad de recuperación, trasladando a estos pacientes a su pieza sólo cuando cumplan los criterios incluidos en la escala de White y Song.

Palabras clave: obesidad; perioperatorio; cirugía; consideraciones. 


\section{Introducción}

La obesidad se define como una condición de acumulación anormal o excesiva de grasa en el tejido adiposo, a un nivel que resulta perjudicial para la salud. La prevalencia de sobrepeso y obesidad está aumentando significativamente en todo el mundo, considerándose una verdadera pandemia. Según estadísticas sanitarias del 2009, en el año 2005 había 1.600 millones de adultos con sobrepeso y 400 millones de adultos obesos. En el Reino Unido, 24\% de los hombres y $25 \%$ de las mujeres son obesos, y más del 3\% de la población tiene obesidad mórbida. Chile ocupa el segundo lugar en Latinoamérica con los más altos índices de obesidad. Estudios locales arrojan que más del $85 \%$ de los chilenos son sedentarios y dos tercios de los adultos presentan obesidad o sobrepeso. En nuestro país la obesidad varía según sexo y nivel educacional, siendo mayor en mujeres que en hombres ( $25 \%$ versus $19 \%$, respectivamente), así como en adultos de bajo nivel socioeconómico ${ }^{1}$. La obesidad es factor de riesgo para el desarrollo de patologías médicas y complicaciones perioperatorias ${ }^{2}$. En la Tabla 1 se muestra la clasificación de la obesidad, según la Organización Mundial de la Salud ${ }^{3}$.

Dado la alta prevalencia de obesidad a nivel mundial, cirujanos y anestesiólogos se enfrentarán cada vez con mayor frecuencia a pacientes obesos que se someterán a procedimientos quirúrgicos. Dadas las múltiples complicaciones asociadas a la obesidad, es fundamental realizar un adecuado manejo perioperatorio de estos pacientes con la finalidad de disminuir la morbimortalidad de esta población quirúrgica. El objetivo de esta revisión es entregar recomendaciones respecto del manejo perioperatorio de pacientes obesos.

\section{Manejo preoperatorio}

\section{Estratificación de riesgo}

La mayoría de los pacientes obesos que se presentan a cirugía no presentan patología crónica significativa y su riesgo perioperatorio es similar al de pacientes normopesos ${ }^{4}$. Los pacientes obesos con mayor riesgo de complicaciones perioperatorias son aquellos con obesidad central y/o síndrome metabólico ${ }^{4}$, debiendo tener especial cuidado en aquellos pacientes con riesgo de síndrome de apnea-hipoapnea obstructiva del sueño (SAHOS) y trombosis venosa profunda (TVP).

Se ha validado el Obesity Surgery Mortality Risk Stratification Score (OS-MRS) con el objetivo de identificar factores de riesgo asociados a mortalidad perioperatoria en aquellos pacientes obesos que serán sometidos a cirugía de bypass gástrico ${ }^{5}$. En la Tabla 2 se detallan los parámetros contenidos en el OS-MRS. En la Tabla 3 se muestra el riesgo de mortalidad perioperatoria según puntaje obtenido en el OS-MRS. Aunque este score está validado sólo para cirugía bariátrica, pero podría extrapolarse a otros procedimientos quirúrgicos ${ }^{3}$. Los pacientes obesos con un puntaje de 4 a 5 puntos en la escala OS-MRS tienen un riesgo de mortalidad de 2,4-3\% $\mathrm{y}$, por tanto, requieren una monitorización perioperatoria más estrecha.

Tabla 1. Clasificación de la Organización Mundial de la Salud de estado nutricional según $\mathrm{IMC}^{3}$

\begin{tabular}{|cc|}
\hline Índice de masa corporal $\left.\mathbf{( k g} / \mathbf{m}^{\mathbf{2}}\right)$ & Clasificación \\
\hline$<18,5$ & Bajo peso \\
$18,5-24,9$ & Normal \\
$25-29,9$ & Sobrepeso \\
$30-34,9$ & Obesidad nivel 1 \\
$35-39,9$ & Obesidad nivel 2 \\
$>40$ & Obesidad nivel 3 \\
\hline
\end{tabular}

Tabla 2. Factores de riesgo asociados a mortalidad perioperatoria según el Obesity surgery mortality risk stratification score (OS-MRS) ${ }^{5}$

\begin{tabular}{|lc|}
\hline Factor de riesgo & Puntaje \\
\hline IMC $>50$ & 1 \\
Hombre & 1 \\
Edad > 45 años & 1 \\
Hipertensión arterial & 1 \\
Factores de riesgo de TVP: & 1 \\
- Tromboembolismo venoso previo & \\
- Usuario de filtro de vena cava & \\
- Trastorno respiratorio del sueño & \\
- Hipertensión pulmonar & \\
\hline
\end{tabular}

Tabla 3. Clasificación según puntaje en el OS-MRS y riesgo de mortalidad perioperatoria ${ }^{5}$

\begin{tabular}{|cc|}
\hline Clasificación & Riesgo de mortalidad \\
\hline Clase A: $0-1$ puntos & $0,2-0,3 \%$ \\
\hline Clase B: $2-3$ puntos & $1,1-1,5 \%$ \\
\hline Clase C: $4-5$ puntos & $2,4-3 \%$ \\
\hline
\end{tabular}




\section{Evaluación respiratoria}

La obesidad provoca una reducción de la capacidad residual funcional (CRF) que aumenta el riesgo de presentar atelectasias y cortocircuitos (shunts) pulmonares perioperatorios ${ }^{6}$. Además, esta patología incrementa la tasa metabólica basal, el trabajo respiratorio y la demanda de oxígeno, provocando que durante un periodo de apnea el nivel de oxígeno arterial disminuya rápidamente, lo que requiere un manejo de vía aérea lo más expedito posible.

Es esencial buscar la presencia de trastornos respiratorios del sueño. Para ello, el cuestionario STOP-BANG (Tabla 4) ha demostrado ser de mayor validez en pacientes obesos ${ }^{7}$. Una puntuación $\geq 5$ puntos indica un riesgo significativo de complicaciones cardiovasculares y respiratorias perioperatorias, sugiriéndose derivar estos pacientes a un médico especialista ${ }^{8}$. En pacientes con historia de marcada disnea de esfuerzos, cefaleas matutinas y electrocardiograma (ECG) con signos de hipertrofia auricular derecha, se puede sospechar la presencia de SAHOS, aun con una baja puntuación STOP-BANG ( $<3$ puntos), recomendándose también derivar a estos pacientes.

Los pacientes con SAHOS no diagnosticada o aquellos que no toleran el CPAP (continue positive airway pressure), presentan mayor riesgo de complicaciones respiratorias perioperatorias y morbilidad cardiovascular, mientras que los pacientes que cumplen cabalmente con el uso del CPAP tienen un riesgo menor de eventos adversos perioperatorios ${ }^{9}$. Gaszynski y cols. compararon el uso de CPAP versus oxígeno por cánula nasal en el posoperatorio de bypass gástrico en pacientes obesos, encontrando que la saturación de oxígeno arterial fue significativamente mayor en el grupo usuario de CPAP a los
30 min, 4 y 8 h después del ingreso a la unidad de recuperación ${ }^{10}$. Jensen y cols. compararon el uso preoperatorio de CPAP o BIPAP (bi-level positive airway pressure) versus prescindir de su uso, en pacientes obesos con o sin diagnóstico de SAHOS, sometidos a bypass gástrico con técnica $\mathrm{Y}$ de Roux. No hubo diferencias significativas en la estadía hospitalaria, complicaciones respiratorias ni mortalidad perioperatoria $^{11}$. Finalmente, Huerta y cols. compararon el uso posoperatorio de CPAP en pacientes sometidos a bypass gástrico versus no usarlo. No hubo correlación entre la utilización de CPAP y la incidencia de dehiscencia de anastomosis, con un riesgo significativamente menor de complicaciones respiratorias posoperatoria ${ }^{12}$. Recomendamos utilizar CPAP en el posoperatorio de pacientes obesos con antecedentes de SAHOS sometidos a cirugía bariátrica.

\section{Vía aérea}

La obesidad se asocia a un $30 \%$ más de probabilidades de presentar intubación difícil con respecto a pacientes normopesos ${ }^{13}$. Una circunferencia de cuello $\geq 60 \mathrm{~cm}$ se asocia a un $35 \%$ de probabilidad de laringoscopia difícil. Además, los pacientes obesos presentan un riesgo aumentado de ventilación difícil con bolsa-mascarilla facial respecto de aquellos normopesos ${ }^{14}$. La presencia de barba también es un predictor de ventilación difícil ${ }^{15}$. Por esto, se sugiere que pacientes obesos se afeiten y/o recorten significativamente su barba en el preoperatorio, con el objetivo de disminuir este riesgo.

\section{Evaluación cardiovascular}

Los componentes del síndrome metabólico deben ser identificados de forma activa en el periodo

Tabla 4. Cuestionario STOP-BANG para el diagnóstico de SAHOS y evaluación del riesgo perioperatorio ${ }^{7}$

\begin{tabular}{|c|c|c|}
\hline Acrónimo STOP-BANG & Cuestionario & Puntaje \\
\hline Snoring & ¿Ronca usted fuerte (más fuerte que lo que habla o se escucha a través de una puerta cerrada)? & 1 \\
\hline Tired & $\begin{array}{l}\text { ¿Se siente usted frecuentemente cansado, fatigado o somnoliento durante el día? ¿Se queda usted } \\
\text { dormido durante el día? }\end{array}$ & 1 \\
\hline Observed & ¿Lo ha observado alguien dejar de respirar, ahogarse o boquear durante el sueño? & 1 \\
\hline Blood Pressure & ¿Es usted hipertenso o ha recibido tratamiento por presión arterial elevada? & 1 \\
\hline$B M I$ & $\mathrm{IMC}>35 \mathrm{~kg} / \mathrm{m}^{2}$ & 1 \\
\hline Age & Edad $>50$ años & 1 \\
\hline Neck & $\begin{array}{l}\text { Circunferencia de cuello (medida alrededor de la manzana de Adán) }>43 \mathrm{~cm} \text { en hombres o }>41 \mathrm{~cm} \\
\text { en mujeres }\end{array}$ & 1 \\
\hline Gender & Género masculino & 1 \\
\hline
\end{tabular}


preoperatorio de pacientes obesos, ya que existe una fuerte asociación con la morbilidad cardiaca perioperatoria ${ }^{16}$. El test de esfuerzo cardiopulmonar puede predecir aquellos pacientes con alto riesgo de complicaciones cardiopulmonares posoperatorias y mayor estadía hospitalaria ${ }^{17}$.

\section{Manejo intraoperatorio}

\section{Preparación del personal y equipamiento}

Para el paciente obeso debe incluirse un equipamiento adecuado, incluyendo mesas quirúrgicas que soporten más de $200 \mathrm{~kg}$ de peso, camillas adecuadas, y equipos de posicionamiento y deslizamiento de estos pacientes ${ }^{3}$.

La experiencia, tanto del anestesiólogo como del cirujano, debe ser considerada. En aquellos pacientes con un score OS-MRS de 4-5 puntos se recomiendan que sean manejados por un anestesiólogo con experiencia en pacientes obesos. Un cirujano con experiencia podría reducir significativamente el tiempo operatorio, lo que ayudará a disminuir la morbilidad perioperatoria ${ }^{3}$.

Deben utilizarse también equipos de monitorización y ventilación adecuados al peso del paciente: manguito de presión arterial no invasiva (PANI) que se ajuste al brazo del paciente, monitores de bloqueo neuromuscular, cánulas orofaríngeas $\mathrm{n}^{\circ} 6 \mathrm{y}$ nasofaríngeas $\mathrm{N}^{\circ} 8$, ventilación mecánica con PEEP (positive end expiratory pressure), etc. ${ }^{3}$

\section{Anestesia regional}

En caso de riesgo aumentado en el manejo de vía aérea de pacientes obesos, la anestesia regional debería preferirse por sobre la general, pero siempre teniendo un plan para el manejo de ésta ${ }^{18}$. Existe un mayor riesgo de fracaso con las técnicas regionales en el paciente obeso, por lo cual se sugiere una adecuada consejería y asesoramiento previo al procedimiento ${ }^{19}$. Equipamiento especial, como agujas espinales o epidurales extra largas, deben estar disponibles. El ultrasonido es una herramienta útil en aquellos procedimientos anestésicos regionales que resulten complejos en estos pacientes $^{20}$.

\section{Inducción en anestesia general}

En general los accesos venosos son más difíciles de instalar en pacientes obesos, por lo que es aconsejable colocar la vía intravenosa en pabellón. El ultrasonido puede ser útil para ayudar a localizar las venas periféricas. Deben considerarse sitios inusuales para los accesos intravenosos, como la parte superior del brazo y la pared anterior del tórax. El acceso venoso central sólo debe utilizarse si el acceso periférico es imposible de instalar o si está indicado específicamente ${ }^{3}$.

Las drogas anestésicas de elección para pacientes obesos son aquellas fácilmente reversibles, con rápido inicio de acción y metabolización. La dosis de las drogas a utilizarse en estos pacientes debe calcularse en base al peso ajustado o peso magro. El peso magro corresponde al peso del paciente excluyendo la grasa corporal. Las fórmulas para calcular estos parámetros se detallan a continuación en la Tabla $5^{3}$.

Dependiendo del tipo de droga, debe utilizarse el peso ajustado o peso magro para calcular la dosis correcta (Tabla 6) ${ }^{3}$.

El National Audit Project 4 (NAP4) analizó las complicaciones del manejo de vía aérea que deben tenerse en cuenta en el paciente obeso, las cuales se detallan en la Tabla $7^{21}$.

Dado que el trabajo respiratorio está aumentado en el paciente obeso, la intubación orotraqueal con ventilación controlada es la técnica de elección para el manejo de vía aérea. El uso de dispositivos supraglóticos debe reservarse para pacientes muy seleccionados, que se someterán a procedimientos quirúrgicos abreviados y que puedan mantener la cabeza erguida durante la cirugía. Además, la vía aérea superior debe estar accesible durante el procedimiento quirúrgico, con un plan anestésico para la intubación orotraqueal si fuese necesaria ${ }^{3}$.

Durante la inducción anestésica, el paciente debe ser colocado en posición de "rampa", donde el trago del pabellón auricular debe estar alineado con el esternón, ya que de esta forma se mejora significativamente la visualización de las cuerdas vocales durante la laringoscopia ${ }^{22,23}$. Además, esta posición optimiza la mecánica pulmonar, ayudando así a la oxigenación y ventilación alveolar, lo cual

Tabla 5. Fórmulas para el cálculo de peso ideal, peso ajustado y peso magro, según sexo ${ }^{3}$

\begin{tabular}{|llll|}
\hline Sexo & Peso ideal & Peso ajustado & Peso magro \\
\hline Masculino & Estatura $(\mathrm{cm})-100$ & Peso ideal $+0,4 *($ peso real - peso ideal $)$ & $(927 *$ peso real $) *(6.680+216 * \text { IMC })^{-1}$ \\
\hline Femenino & Estatura $(\mathrm{cm})-105$ & Peso ideal $+0,4 *($ peso real - peso ideal $)$ & $(927 *$ peso real $) *(8.780+244 * I M C))^{-1}$ \\
\hline
\end{tabular}


Tabla 6. Drogas anestésicas y cálculo de dosis según peso magro o peso ajustado del paciente ${ }^{3}$

\begin{tabular}{|ll|}
\hline Peso magro & Peso ajustado \\
\hline Propofol (inducción) & Propofol (infusión) \\
\hline Tiopental & Antibióticos \\
Fentanilo & Heparinas de bajo peso molecular \\
\hline Rocuronio & Alfentanilo \\
\hline Vecuronio & Neostigmina \\
\hline Atracurio & Sugammadex \\
Morfina & \\
\hline Paracetamol & \\
\hline Lidocaína & \\
Bupivacaína & \\
\hline
\end{tabular}

Tabla 7. Causas de complicaciones en el manejo de vía aérea del paciente obeso (NAP4) ${ }^{21}$

Falta de reconocimiento y planificación de posibles problemas de vía aérea

Reducido tiempo de apnea segura

Alta tasa de fracaso en instalación de dispositivos de vía aérea de rescate, como máscara laríngea y criotirotomía de emergencia

Tabla 8. Estrategias perioperatorias para disminuir el riesgo de complicaciones respiratorias en la población obesa ${ }^{3}$

Evitar la anestesia general y sedantes cuando sea posible

Uso de fármacos de acción corta

Uso de monitores de profundidad anestésica para limitar la cantidad de fármacos al mínimo posible, especialmente gases halogenados y propofol

Uso de monitorización del bloqueo neuromuscular para mantener un nivel de bloqueo compatible con la cirugía y asegurar su completa reversión antes de "despertar" al paciente

Utilización máxima de anestésicos locales y analgesia multimodal para disminuir el consumo de opioides

Mantenimiento de la cabeza hiperextendida durante el despertar

Monitorización de la saturación de oxígeno en el posoperatorio

maximiza el tiempo de apnea seguro. La adición de PEEP puede facilitar aún más la preoxigenación del paciente en esta posición ${ }^{24}$.

Las fasciculaciones asociadas a la succinilcolina aumentan el consumo de oxígeno y acortan el tiempo de apnea segura, por lo que no es el fármaco de elección para el bloqueo muscular de estos pacientes ${ }^{25}$. Últimamente, los aminoesteroides son los bloqueadores neuromusculares de elección en la población obesa debido a la aparición del sugammadex. Esta droga es un antagonista específico de este tipo de bloqueadores neuromusculares. La dosis de sugammadex para la reversión del relajo neuromuscular de emergencia debe ser calculada de antemano y estar disponible de forma inmediata ${ }^{26}$.

El peso ideal debe utilizarse para calcular el tamaño de los tubos orotraqueales y el volumen corriente durante la ventilación controlada. El diámetro traqueal se reduce ligeramente con el aumento de índice de masa corporal ${ }^{27}$. Ningún modo particular de ventilación controlada ha demostrado ser superior en pacientes obesos, sin embargo, mayores volúmenes corrientes para una presión máxima determinada pueden lograrse utilizando la ventilación controlada por presión, en vez de por volumen ${ }^{3}$. La adición de un PEEP suficiente y maniobras de reclutamiento reducen el riesgo de atelectasias intra y posoperato$\operatorname{rias}^{28}$. En cirugía laparoscópica, la flexión del tronco del paciente, es decir, una ligera posición sentada, mejora la excursión abdominal para el cirujano y se traduce en menores presiones de vía aérea ${ }^{29}$.

\section{Trastornos respiratorios del sueño en anestesia}

Un principio simple y seguro es suponer que todos los pacientes obesos tienen algún grado de trastorno respiratorio del sueño. Estrategias útiles para disminuir el riesgo de complicaciones respiratorias en el perioperatorio se muestran en la Tabla $8^{3}$.

\section{Mantención en anestesia general}

En la actualidad disponemos de escasa evidencia para recomendar el uso Target Controlled Infusion (TCI) de propofol o gases halogenados en el mantenimiento de la anestesia en la población obesa. Sin embargo, debido al aumento del riesgo de awareness accidental en obesos, es importante que la mantención anestésica se inicie inmediatamente después de la inducción ${ }^{30}$. Los gases halogenados insolubles en grasas, tales como desflurano o sevoflurano, tienen un inicio y fin de acción más rápido que el isoflurano, y por tanto, se prefieren para la mantención anestésica de estos pacientes. Existe evidencia de recuperación de reflejos de vía aérea más rápida con desflurano versus sevoflurano en obesos $^{31}$. Se recomienda utilizar técnicas de analgesia multimodal, incluyendo anestesia local, bloqueos de nervios periféricos y anestesia peridural, para disminuir los requerimientos de opiáceos sistémicos intra y posoperatorios ${ }^{3}$.

\section{Despertar en anestesia general}

La ausencia de bloqueo neuromuscular residual debe ser objetivada por un estimulador de nervio 
periférico, con el objetivo de asegurar la capacidad ventilatoria (reflejo tusígeno por ejemplo) y protección de la vía aérea previo al despertar ${ }^{30}$. Aparte de haber recuperado los reflejos de vía aérea, los pacientes obesos deben tener ventilación espontánea, con volúmenes corrientes adecuados y frecuencias respiratorias entre 8 y 30 ventilaciones por minuto antes de la extubación traqueal, la cual debe realizarse con el paciente lo más despierto y semisentado posible.

En aquellos pacientes con antecedentes de SAHOS, la inserción de un cánula nasofaríngea antes del despertar anestésico ayuda a mitigar la obstrucción parcial de la vía aérea superior, la cual ocurre comúnmente en la población obesa ${ }^{3}$.

\section{Manejo posoperatorio}

Todo paciente obeso sometido a anestesia y/o sedación debe ingresar a una Unidad de Recuperación Posoperatoria (URP) una vez concluido su procedimiento quirúrgico; monitorizándose como mínimo con electrocardiografía continua, oximetría de pulso, PANI, control de temperatura al ingreso a la Unidad, y medición de frecuencia respiratoria y nivel de conciencia frecuentemente. Se recomienda que los pacientes con SAHOS usuarios de CPAP reinicien el uso de éste en la URP o inmediatamente al volver a su pieza ${ }^{32}$.

La oxigenoterapia es recomendable para mantener los niveles preoperatorios de saturación arterial de oxígeno. Si el paciente requiere oxígeno suplementario, éste puede administrarse por naricera, mascarilla venturi o a través del CPAP.

Antes del alta de la URP, todos los pacientes obesos deben ser evaluados buscando signos de hipoventilación, episodios de apnea o hipopnea y/o desaturación de oxígeno.

El paciente puede ser enviado a su pieza sólo cuando cumpla con los criterios de alta de White $y$ Song, los cuales se detallan en la Tabla $9^{33}$. Se necesita un puntaje $\geq 12$ puntos y ningún parámetro con puntaje de cero para que el paciente pueda ser trasladado. Existen muchos scores de criterios de alta disponibles, pero el de White y Song ha demostrado ser de mayor utilidad.

\section{Analgesia y cuidados en pieza}

Se recomienda mantener al paciente en posición semisentado a $45^{\circ}$. La movilización precoz es vital por lo que se sugiere realizarla incluso el mismo día de la cirugía, lo que implica necesariamente una analgesia apropiada ${ }^{3}$.

Tabla 9. Criterios de alta desde la unidad de recuperación según la escala de White y Song ${ }^{33}$

\begin{tabular}{|llc|}
\hline Parámetro & Descripción & Puntaje \\
\hline Conciencia & Despierto y orientado & 2 \\
& Requiere mínimo estímulo para ser despertado & 1 \\
Hemodinamia & No despierta con estímulo táctil & 0 \\
& PA+/- $15 \%$ del nivel precirugía & 2 \\
& PA+/- 30\% del nivel precirugía & 1 \\
Oximetría & PA+/- 50\% del nivel precirugía & 0 \\
& Saturación $>92 \%$ aire ambiente & 2 \\
& Requerimientos de oxígeno para Saturación $>90 \%$ & 1 \\
Respiración & Saturación <90\% a pesar de oxigenoterapia & 0 \\
& Respira profundamente y tose, sin taquipnea & 2 \\
Actividad & Taquipnea, pero buena capacidad para toser & 1 \\
& Disnea con mala capacidad para toser & 0 \\
Dolor & Moviliza extremidades & 2 \\
& Debilidad leve-moderada de extremidades & 1 \\
& Incapaz de movilizar extremidades & 0 \\
\hline NVPO & Sin dolor & 2 \\
& Dolor moderado-severo, controlable con analgesia & 1 \\
& Dolor persistente pese a analgesia & 0 \\
\hline
\end{tabular}


El uso de sistemas de analgesia controlada por el paciente o patient controlled analgesia (PCA) en base a opioides endovenosos requiere una cuidadosa evaluación, debido al aumento del riesgo de depresión respiratoria en pacientes con SAHOS. Por otro lado, la PCA peridural se ha asociado con movilidad posoperatoria reducida, lo que podría aumentar el riesgo de fenómenos tromboembólicos posoperatorios en estos pacientes ${ }^{3}$.

En caso de requerirse, la oxigenoterapia debe continuar hasta que se alcancen las saturaciones de oxígeno basales del paciente. La oximetría de pulso debe monitorizarse frecuentemente hasta que el paciente se encuentre sin requerimientos de oxígeno suplementario ni con opiáceos parenterales ${ }^{3}$.

\section{Tromboprofilaxis}

La obesidad es un factor de riesgo per se de fenómenos tromboembólicos, recomendándose que todos los pacientes obesos reciban algún tipo de profilaxis de TVP, independiente del tipo de procedimiento quirúrgico ${ }^{3}$. Las estrategias para reducir el riesgo de eventos tromboembólicos incluyen: adecuada posición del paciente en la mesa quirúrgica, compresión neumática intermitente, medias antitrombóticas, movilización posoperatoria precoz, uso de fármacos anticoagulantes perioperatorios y filtros de vena cava inferior ${ }^{34}$. La evidencia reciente no apoya el uso de rutina de filtros de vena cava inferior como tromboprofilaxis en la población obe$\mathrm{sa}^{35}$. Durante la cirugía, los pacientes deben tener las rodillas flexionadas en $15^{\circ}$, con un "rollo" de sábanas superior al hueco poplíteo, taloneras (para protección de puntos de apoyo), medias antitrombóticas elasticadas en extremidades inferiores (hasta el muslo) y compresión neumática intermitente.

El pilar de la profilaxis de TVP en la población obesa es farmacológico, siendo las heparinas de bajo peso molecular (HBPM) los fármacos anticoagulantes más utilizados. La Tabla 10 muestra las dosis profilácticas posoperatorias de las HBPM más usadas actualmente, ajustadas por peso del paciente ${ }^{34}$.

Tabla 10. Dosis de profilaxis de TVP de las HBPM, ajustadas por peso del paciente ${ }^{34}$

\begin{tabular}{|ccc|}
\hline Peso paciente (kg) & Enoxaparina & Dalteparina \\
$<50$ & $20 \mathrm{mg}$ al día & $2.500 \mathrm{U}$ al día \\
$50-100$ & $40 \mathrm{mg}$ al día & $5.000 \mathrm{U}$ al día \\
$100-150$ & $40 \mathrm{mg}$ cada $12 \mathrm{~h}$ & $5.000 \mathrm{U}$ cada $12 \mathrm{~h}$ \\
$>150$ & $60 \mathrm{mg}$ cada $12 \mathrm{~h}$ & $7.500 \mathrm{U}$ cada $12 \mathrm{~h}$ \\
\hline
\end{tabular}

Los nuevos anticoagulantes orales, como rivaroxabán y dabigatrán, están aprobados para la profilaxis de TVP posterior a cirugías traumatológicas, sin embargo, la evidencia aún es limitada para recomendar su uso en la población obesa en otro tipo de cirugías ${ }^{34}$.

\section{Conclusiones}

La obesidad se define como una condición de acumulación excesiva de grasa corporal a un nivel que resulta dañino para la salud. La prevalencia del sobrepeso y obesidad está aumentando en todo el mundo a un ritmo alarmante, considerándose una verdadera pandemia. Es un importante factor de riesgo para el desarrollo de patologías médicas y complicaciones perioperatorias.

La gran mayoría de pacientes obesos que se someten a procedimientos quirúrgicos tienen poca patología agregada, por lo que su riesgo perioperatorio es similar a pacientes normopesos. El OS-MRS es una escala validada para evaluar el riesgo de mortalidad perioperatoria de la población obesa. Aquellos pacientes con un puntaje de 4 ó 5 puntos requieren una monitorización quirúrgica mucho más estricta.

La presencia de SAHOS debe buscarse dirigidamente en estos pacientes, utilizando el cuestionario STOP-BANG, el cual tiene mayor validez en la población obesa. Una puntuación STOP-BANG $\geq 5$ indica una alta probabilidad de esta patología.

Durante la inducción anestésica se recomienda colocar al paciente en posición "de rampa", ya que mejora significativamente la visualización de la glotis durante la laringoscopia, además de optimizar la oxigenación y ventilación, lo cual aumenta el tiempo de apnea seguro. Dado que el trabajo respiratorio está aumentado en el paciente obeso, la intubación orotraqueal con ventilación controlada es la técnica de elección para el manejo de vía aérea durante la cirugía.

Las dosis de drogas anestésicas a utilizarse en estos pacientes deben calcularse en base al peso ajustado o magro, dependiendo del tipo de fármaco elegido.

Una monitorización posoperatoria completa y frecuente debe mantenerse en la URP en los pacientes obesos, trasladándose a su pieza sólo cuando presenten un puntaje $\geq 12$ puntos según la escala de White y Song (con ningún ítem en cero puntos).

El objetivo de esta revisión fue definir y detallar las consideraciones perioperatorias más relevantes a tener en cuenta en pacientes obesos, con el fin de establecer, según la mejor evidencia disponible en 
la literatura, recomendaciones actualizadas y resumidas al equipo quirúrgico tratante.

\section{Responsabilidades éticas}

Protección de personas y animales. Los autores declaran que para esta investigación no se han realizado experimentos en seres humanos ni en animales.

Confidencialidad de los datos. Los autores declaran que en este artículo no aparecen datos de pacientes.

Derecho a la privacidad y consentimiento informado. Los autores declaran que en este artículo no aparecen datos de pacientes.

\section{Conflicto de intereses}

Los autores no refieren conflictos de interés.

\section{Referencias}

1. Vio F, Albala C, Kain J. Nutrition transition in Chile revisited: mid-term evaluation of obesity goals for the period 2000-2010. Public Health Nutr. 2008;11:405-12.

2. Obesity: preventing and managing the global epidemic. Report of a WHO consultation. World Health Organ Tech Rep Ser. 2000;894:i-xii, 1-253.

3. Nightingale CE, Margarson MP, Shearer E, Redman JW, Lucas DN, Cousins JM, et al. Peri-operative management of the obese surgical patient 2015: Association of Anaesthetists of Great Britain and Ireland Society for Obesity and Bariatric Anaesthesia. Anaesthesia 2015;70:859-76.

4. Glance LG, Wissler R, Mukamel DB, Li Y, Diachun CA, Salloum R, et al. Perioperative outcomes among patients with the modified metabolic syndrome who are undergoing noncardiac surgery. Anesthesiology 2010;113:859-72.

5. DeMaria EJ, Murr M, Byrne TK, Blackstone R, Grant JP, Budak A, et al. Validation of the obesity surgery mortality risk score in a multicenter study proves it stratifies mortality risk in patients undergoing gastric bypass for morbid obesity. Ann Surg. 2007;246:578-82; discussion 83-4.

6. Pelosi P, Croci M, Ravagnan I, Tredici S, Pedoto A, Lissoni A, et al. The effects of body mass on lung volumes, respiratory mechanics, and gas exchange during general anesthesia. Anesth Analg. 1998;87:654-60.

7. Chung F, Subramanyam R, Liao P, Sasaki E, Shapiro C, Sun Y. High STOP-Bang score indicates a high probability of obstructive sleep apnoea. Br J Anaesth. 2012;108:768-75.

8. Chung F, Yang Y, Liao P. Predictive performance of the STOP-Bang score for identifying obstructive sleep apnea in obese patients. Obes Surg. 2013;23:20507.

9. Hallowell PT, Stellato TA, Petrozzi MC, Schuster M, Graf K, Robinson A, et al. Eliminating respiratory intensive care unit stay after gastric bypass surgery. Surgery. 2007;142:608-12; discussion 12.e1.

10. Gaszynski T, Tokarz A, Piotrowski D, Machala W. Boussignac CPAP in the postoperative period in morbidly obese patients. Obes Surg. 2007;17:452-6.

11. Jensen C, Tejirian T, Lewis C, Yadegar J, Dutson E, Mehran A. Postoperative CPAP and BiPAP use can be safely omitted after laparoscopic Roux-en-Y gastric bypass. Surg Obes Relat Dis. 2008;4:512-4.

12. Huerta S, DeShields S, Shpiner R, Li Z, Liu C, Sawicki M, et al. Safety and efficacy of postoperative continuous positive airway pressure to prevent pulmonary complications after Rouxen-Y gastric bypass. J Gastrointest Surg. 2002;6:354-8.

13. Lundstrøm LH, Møller AM, Rosenstock C, Astrup G, Wetterslev J. High body mass index is a weak predictor for difficult and failed tracheal intubation: a cohort study of 91,332 consecutive patients scheduled for direct laryngoscopy registered in the Danish Anesthesia Database. Anesthesiology 2009;110:26674.

14. Kheterpal S, Martin L, Shanks AM, Tremper KK. Prediction and outcomes of impossible mask ventilation: a review of 50,000 anesthetics. Anesthesiology 2009;110:891-7.
15. Langeron $\mathrm{O}$, Masso E, Huraux $\mathrm{C}$, Guggiari M, Bianchi A, Coriat $\mathrm{P}$, et al. Prediction of difficult mask ventilation. Anesthesiology 2000;92:1229-36.

16. Apovian CM, Gokce N. Obesity and cardiovascular disease. Circulation 2012;125:1178-82

17. Hennis PJ, Meale PM, Hurst RA, O'Doherty AF, Otto J, Kuper M, et al. Cardiopulmonary exercise testing predicts postoperative outcome in patients undergoing gastric bypass surgery. $\mathrm{Br} \mathrm{J}$ Anaesth. 2012;109:566-71.

18. Nielsen KC, Guller U, Steele SM, Klein SM, Greengrass RA, Pietrobon R. Influence of obesity on surgical regional anesthesia in the ambulatory setting: an analysis of 9,038 blocks. Anesthesiology 2005;102:181-7.

19. Ingrande J, Brodsky JB, Lemmens HJ. Regional anesthesia and obesity. Curr Opin Anaesthesiol. 2009;22:683-6.

20. Balki M, Lee Y, Halpern S, Carvalho JC Ultrasound imaging of the lumbar spine in the transverse plane: the correlation between estimated and actual depth to the epidural space in obese parturients. Anesth Analg 2009;108:1876-81.

21. Cook TM, Woodall N, Frerk C, Project FNA. Major complications of airway management in the UK: results of the Fourth National Audit Project of the Royal College of Anaesthetists and the Difficult Airway Society. Part 1: anaesthesia. Br J Anaesth. 2011;106:61731.

22. Mhyre JM. Anesthetic management for the morbidly obese pregnant woman. Int Anesthesiol Clin. 2007;45:51-70.

23. Collins JS, Lemmens HJ, Brodsky JB, Brock-Utne JG, Levitan RM. Laryngoscopy and morbid obesity: a 
comparison of the "sniff" and "ramped" positions. Obes Surg. 2004; 14:1171-5.

24. Gander S, Frascarolo P, Suter M, Spahn DR, Magnusson L. Positive endexpiratory pressure during induction of general anesthesia increases duration of nonhypoxic apnea in morbidly obese patients. Anesth Analg. 2005;100:580-4.

25. Taha SK, El-Khatib MF, Baraka AS, Haidar YA, Abdallah FW, Zbeidy RA, et al. Effect of suxamethonium $v s$ rocuronium on onset of oxygen desaturation during apnoea following rapid sequence induction. Anaesthesia. 2010;65:358-61.

26. Bisschops MM, Holleman C, Huitink JM. Can sugammadex save a patient in a simulated 'cannot intubate, cannot ventilate' situation? Anaesthesia 2010;65:936-41.

27. D’Anza B, Knight J, Greene JS. Does body mass index predict tracheal airway size? Laryngoscope 2015;125:1093-7.

28. Santesson J. Oxygen transport and venous admixture in the extremely obese. Influence of anaesthesia and artificial ventilation with and without positive endexpiratory pressure. Acta Anaesthesiol Scand. 1976;20:387-94.

29. Mulier JP, Dillemans B, Van Cauwenberge S. Impact of the patient's body position on the intraabdominal workspace during laparoscopic surgery. Surg Endosc. 2010;24:1398-402.

30. Pandit JJ, Andrade J, Bogod DG, Hitchman JM, Jonker WR, Lucas N, et al. The 5th National Audit Project (NAP5) on accidental awareness during general anaesthesia: protocol, methods and analysis of data. Anaesthesia 2014;69:1078-88

31. McKay RE, Malhotra A, Cakmakkaya OS, Hall KT, McKay WR, Apfel CC. Effect of increased body mass index and anaesthetic duration on recovery of protective airway reflexes after sevoflurane $v s$ desflurane. $\mathrm{Br}$ J Anaesth. 2010;104:175-82.

32. Apnea ASoATFoPMopwos. Practice guidelines for the perioperative management of patients with obstructive sleep apnea: an updated report by the American Society of Anesthesiologists Task Force on Perioperative Management of patients with obstructive sleep apnea. Anesthesiology 2014;120:268-86.

33. White PF, Song D. New criteria for fast-tracking after outpatient anesthesia: a comparison with the modified Aldrete's scoring system. Anesth Analg. 1999;88:1069-72.

34. Anakwe RE, Middleton SD, BeresfordCleary N, McEachan JE, Talwalkar SC. Preventing venous thromboembolism in elective upper limb surgery. J Shoulder Elbow Surg. 2013;22:432-8.

35. Rowland SP, Dharmarajah B, Moore HM, Lane TR, Cousins J, Ahmed $\mathrm{AR}$, et al. Inferior vena cava filters for prevention of venous thromboembolism in obese patients undergoing bariatric surgery: a systematic review. Ann Surg. 2015;261:35-45. 